\title{
Suicide in Parkinson's disease
}

\author{
Melissa Deanna Shepard, ${ }^{1}$ Kate Perepezko, ${ }^{1}$ Martijn P G Broen, ${ }^{2}$ \\ Jared Thomas Hinkle, ${ }^{1,3}$ Ankur Butala, ${ }^{1,4}$ Kelly A Mills, ${ }^{4,5}$ Julie Nanavati, ${ }^{1}$ \\ Nicole Mercado Fischer, ${ }^{1}$ Paul Nestadt, ${ }^{1}$ Gregory Pontone ${ }^{1,5}$
}

- Additional material is

published online only. To view please visit the journal online (http://dx.doi.org/10.1136/ jnnp-2018-319815).

${ }^{1}$ Department of Psychiatry and Behavioral Sciences, Johns Hopkins University School of Medicine, Baltimore, Maryland, USA

${ }^{2}$ Department of Neurology, Maastricht University Medical Centre, Maastricht, The

Netherlands

${ }^{3}$ Medical Scientist Training

Program, Johns Hopkins

School of Medicine, Baltimore, Maryland, USA

${ }^{4}$ Department of Neurology, Johns Hopkins University

School of Medicine, Baltimore, Maryland, USA

${ }^{5}$ Morris K. Udall Parkinson's Disease Research Center of Excellence, Johns Hopkins University School of Medicine, Baltimore, Maryland, USA

\section{Correspondence to}

Dr Melissa Deanna Shepard, Department of Psychiatry and Behavioral Sciences, Johns Hopkins University School of Medicine, Baltimore, MD 21205 , USA; mshepar6@jhmi.edu

Received 11 October 2018 Revised 24 December 2018 Accepted 27 December 2018 Published Online First 19 January 2019

Check for updates

(C) Author(s) (or their employer(s)) 2019. No commercial re-use. See rights and permissions. Published by BMJ.

To cite: Shepard MD,

Perepezko K, Broen MPG,

et al. J Neurol Neurosurg

Psychiatry 2019;90:822-829.

\section{ABSTRACT}

Persons with Parkinson's disease (PwP) have many known risk factors for suicide and suicidal ideation (SI). Despite this, there is limited understanding of suicidality in this population. We conducted a systematic review to synthesise the available literature on suicidality in PwP and highlight areas for potential intervention and further research. We identified 116 articles discussing SI, suicidal behaviours, suicide attempts and/or fatal suicide in PwP. These articles describe prevalence, suicide methods, risk factors for suicide and SI and treatment of suicidality. In this review, we summarise the current literature and provide suggestions for how clinicians can identify and treat PwP who are at risk for suicide, for example, through aggressive treatment of depression and improved screening for access to lethal means.

\section{INTRODUCTION}

Parkinson's disease (PD) is estimated to occur in 1903 per 100000 individuals over age 80 worldwide, and rates will likely continue to increase as the population ages. ${ }^{1} \mathrm{PD}$ is primarily diagnosed by the presence of motor symptoms including bradykinesia, resting tremor and muscle rigidity. ${ }^{2}$ However, non-motor symptoms of PD are increasingly recognised as a cause of poorer quality of life, ${ }^{3}$ nursing home placement ${ }^{4}$ and as predictors of overall mortality ${ }^{5}$ in PD.

Suicide is a feared outcome in psychiatry. Suicide is consistently listed among the top 10 causes of death in the USA, with rates steadily climbing throughout the 21st century and reaching an annual rate of 13.4 deaths per 100000 in $2016 .{ }^{67}$ It is a dominant cause of mortality among the elderly ${ }^{8}$ and persons with neurological diseases including stroke, ${ }^{9}$ Huntington's disease ${ }^{10}$ and epilepsy. ${ }^{11}$ Suicide is a behavioural outcome with a complex aetiology. In the general population and among elderly individuals, depression is a major risk factor for suicide. ${ }^{12}$ However, suicide cannot be understood merely as the most extreme manifestation on the spectrum of depressive disorders. Other identified risk factors for suicide in the general population include substance dependence, access to firearms, anxiety, insomnia and physical disability. ${ }^{13-15}$ Researchers have used this knowledge to develop ways to treat those at risk for suicide. ${ }^{16}$

Persons with PD (PwP) have many risk factors for suicide. Depression in particular is common in PD, with one meta-analysis suggesting that $17 \%$ of PwP suffer from major depressive disorder and $35 \%$ suffer from clinically significant depressive symptoms. ${ }^{17}$ Depressive symptoms in PwP are often phenomenologically distinct from those seen in the general population, for example, PwP may report less guilt, less anhedonia, less sadness, better self-attitude and more dissatisfaction. ${ }^{18}{ }^{19}$ These differences in depressive phenotypes may portend a different risk of suicide in PD as compared with the general population. Insomnia ${ }^{20}$ and anxiety ${ }^{21}$ are also common in PwP and may affect their risk for suicide.

Despite these risk factors, there is little consensus on the association between suicide and PD. In the past, conventional wisdom suggested that suicide rates may be lower in PwP than in the general population but studies over the past few decades have been conflicting, thus justifying an up-todate review of the literature. Scoping reviews are designed to provide an overview of the current state of the literature, contextualise what is known about a subject thus far and identify areas for future research. ${ }^{22}$ Here, we attempt to meet these goals with the aim of better understanding risk factors, mechanisms and prevention of suicide in PwP.

\section{METHODS}

A search of the literature was conducted using Medline (PubMed), Embase, The Cochrane Library (Cochrane Database of Systematic Reviews, Cochrane Central Register of Controlled Trials (CENTRAL), Cochrane Methodology Register), PsycINFO (Ebsco) and Web of Science (Science and Social Science Citation Index). No restrictions were placed on the publication dates or language. For the search strategies designed for Medline (PubMed), the Cochrane Library, Embase and PsycINFO, controlled vocabulary terms for each concept were identified and combined with keyword synonyms. Web of Science was searched using keyword terms only. A handful of studies were known prior to conducting this literature search; they were used to validate the veracity of the search technique. Additional methods for finding articles included searching key journals, the references of the identified articles and the grey literature. See online supplementary appendix 1 for detailed search strategy.

Two reviewers (MS, KP) independently excluded articles based on title and abstract screening. Conflicts were resolved through discussion or a third reviewer's (MB) evaluation. Included articles underwent independent full text evaluation, again by two authors (MS, KP). Inclusion criteria for title and abstract screening as well as full text review 


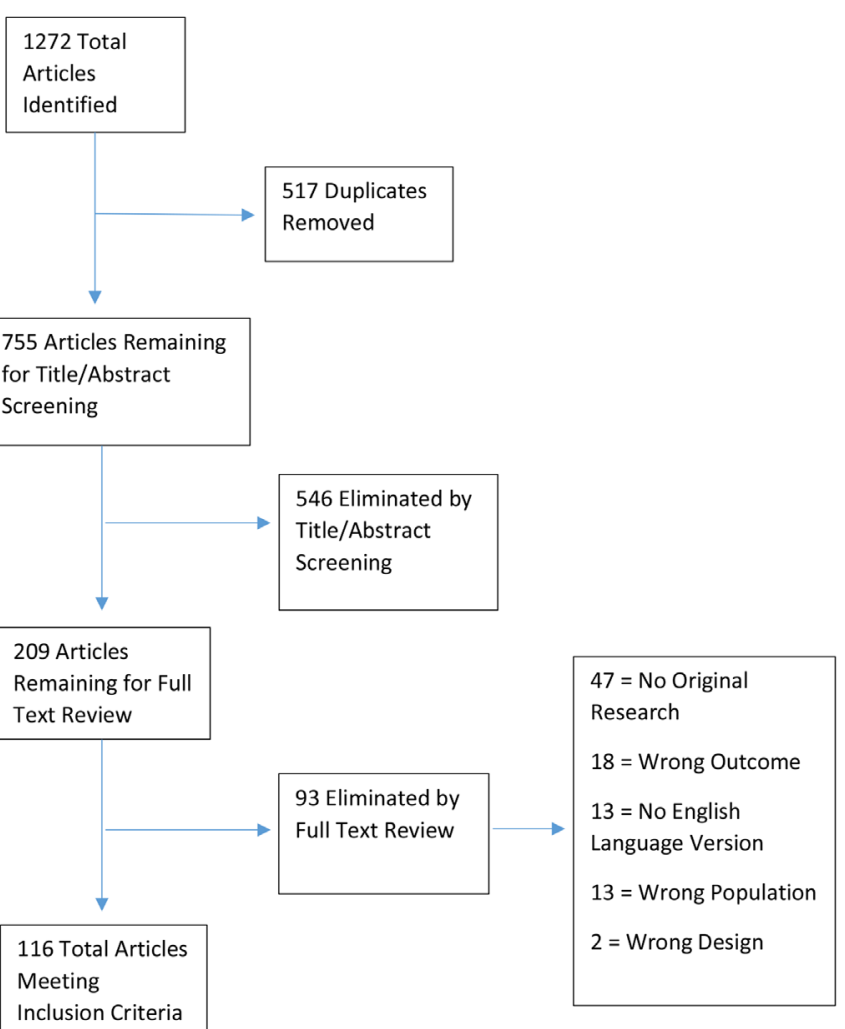

Figure 1 Flow diagram of review process.

were: includes individuals with idiopathic PD; discusses suicidal ideation (SI), suicidal behaviours including suicide attempts (SAs) and/or fatal suicide. Articles were excluded if they were not available in English or did not include original research, such as meta-analyses and review articles. Conflicts were again resolved by discussion or adjudication by a third reviewer (MB). See figure 1 for flow diagram.

We assessed study quality using the Grading of Recommendations Assessment, Development and Evaluation (GRADE) criteria. This strategy is used to evaluate an evidence base for the purposes of deriving guidelines and expressing confidence in recommendations. We used the core tenets of the GRADE criteria, but modified our approach as described below to focus on a summarisation of available literature as opposed to the creation of recommendations. ${ }^{23}$
The quality of each study was reviewed by MS. Each study was rated on a scale from 0 to 5 , with 5 representing the highest quality and 0-1 representing very low quality. Randomised trials were initially assigned a high quality (4), observational studies were initially assigned a low quality rating (2) and case series/case reports were initially assigned a very low quality. A point was added for larger study size (defined as greater than 100 participants). A point was subtracted if there was high risk of bias or limited information available, such as in the case of abstracts or letters to the editor. ${ }^{23}$ See figure 2 for details on this approach. Ratings were compiled in online supplementary appendix 2 under the 'Quality' header. Any comments on the quality assessment for an individual article were included under the 'Comments' header.

\section{RESULTS}

A total of 1269 articles were identified through our database search. Three articles were added after being identified through other sources as described in the Methods section. Two-hundred and nine articles remained after title and abstract screening. After full text review, 116 articles met inclusion criteria. Articles fell into the following categories: prevalence studies, comparison of risk to the general population and other diseases, suicide methods, anatomy and biomarkers, risk factors for suicide and treatments for suicidality. Studies falling into more than one category were included in each. See online supplementary appendix 2 for a detailed description and summary of the relevant findings from each of the articles reviewed. See online supplementary appendix 3 for the references of each of the 116 articles meeting inclusion criteria (denoted with superscripts s1 through s116).

\section{Prevalence and risk of attempted and fatal suicide}

Twenty-one articles discussed prevalence and risk of attempted or fatal suicide in PwP. All of these studies were observational and all were rated as low or moderate quality. The first paper to report on suicide in PD was the 1967 article by Hoehn and Yahr, which described causes of mortality in parkinsonism. Among 340 patients with Parkinson's disease who died during the study period, three died by suicide. ${ }^{\text {s1 }}$ Importantly, there was little distinction between idiopathic and atypical parkinsonian syndromes at that time, and the extent to which this contributed to the suicide rate is unclear.

Most of the research reviewed suggested an elevated suicide risk in PwP. ${ }^{\text {s2-s6 }}$ Risk varies by population; for example, a group in South Korea found a standardised mortality ratio for suicide

\begin{tabular}{|l|l|l|l|}
\hline \multicolumn{2}{|l|}{$\begin{array}{l}\text { Initial Study Quality Assignment Based } \\
\text { on Study Design }\end{array}$} & \multicolumn{1}{|l|}{$\begin{array}{l}\text { High risk of } \\
\text { bias/limited } \\
\text { information }=-1\end{array}$} \\
\hline Randomized trial & High (4) & & Final Quality Rating \\
\hline $\begin{array}{l}\text { Observational } \\
\text { study }\end{array}$ & Low (2) & & $\begin{array}{l}\text { Large sample } \\
\text { size }=+1\end{array}$ \\
\hline $\begin{array}{l}\text { Case series/case } \\
\text { report }\end{array}$ & Very Low (1) & & \\
\hline
\end{tabular}

Figure 2 Process for assessment of study quality. Adapted from Balshem et al (2011). Bias and other methodological concerns commonly included imprecisely defining suicide-related constructs, reporting suicide as a secondary outcome or incidental finding, failing to control for an important variable or excluding important information (ie, demographic information, treatment regimen). Letters to the editor or Abstracts were considered to have limited information available, thus a point was deducted. A sample size was considered to be large if it included over 100 participants. 
in PwP of 1.99 (95\% CI 1.33 to 2.85). ${ }^{.4}$ In the Netherlands, the OR for PD was 2.9 (95\% CI 1.8 to 4.6$)$ in those who attempted suicide via poisoning. ${ }^{56}$ Studies of Serbian and British populations have demonstrated a suicide risk over five times that of the general population matched for age and sex. ${ }^{s 5, \mathrm{~s} 7}$ In British Columbia, suicide is the second most common cause of injury-related mortality in PwP. ${ }^{\mathrm{s} 8}$

In contrast, a few studies have found no elevated suicide risk in PwP. In Denmark, Finland, the USA and Canada, suicide rates among PwP are comparable to the general population after adjusting for confounding factors. ${ }^{\text {s9-s12 }}$ Only one study suggested a decreased suicide rate in PwP. Using United States National Center for Health Statistics data $(n=12430$ 473), Myslobodsky et $a l^{513}$ identified a cumulative incidence of suicide 10 times lower in PwP compared with the general population $(0.08 \%, \mathrm{n}=122$ vs $0.8 \%$ ). Cause of death was determined from a computerised death registry, and unknown causes of death were not classified as suicide. One French study found that suicide occurred in 1\% of PwP; however, it is unclear how this relates to the suicide rate in a matched group of the general population. ${ }^{\text {s14 }}$

Unlike deaths, SAs are difficult to accurately measure in large retrospective studies. Several researchers have noted no SAs in their study population. ${ }^{\mathrm{s} 15, \mathrm{~s} 16}$ Others have found that PwP have a lifetime rate of SAs similar to the general population (around $5 \%)^{\text {s17-s } 19,24}$

\section{Prevalence and risk of suicidal ideation (SI)}

There were 12 articles on prevalence and risk of SI in PwP. Four of these were abstracts. Three were rated as very low quality, six as low quality and three as moderate quality. Most found rates of SI among PwP to be around 30\% with outlying estimates as low as $4 \% .^{\mathrm{s} 7, \mathrm{~s} 16, \mathrm{~s} 18-\mathrm{s} 21}$ Three studies have noted rates of SI lower than $15 \%$, with only two others suggesting PwP are at decreased risk of SI compared with the general population. ${ }^{\text {s11,s15,s17,s21,s22 }}$

\section{Suicide methods}

There were nine studies on suicide methods in PwP. Two studies, both of moderate quality, directly compared suicide methods in PwP to those of the general population. Mainio and colleagues ${ }^{\mathrm{s} 10}$ compared characteristics of nine suicide victims with PD to 546 suicide victims over age 50 without PD and found that PwP were more likely to use violent means (such as hanging or firearms). A Canadian study noted that suicide victims with PD $(n=25)$ were more likely to die by suffocation, excluding hanging, as compared with suicide victims in the general population $(n=1304) .{ }^{s 23}$ Suicide by defenestration and poisoning (including drug overdose) have also been reported in PwP. ${ }^{\text {s6,s24-s27 }}$

Notably, physician-assisted death in PwP was largely absent from this review of the literature. Assisted-death was discussed in only two of the articles reviewed. ${ }^{527,528}$ Both received the lowest quality rating, in part due to difficulty discerning number of participants with idiopathic PD versus other movement disorders.

\section{Anatomy, physiology and biomarkers}

In the general population, several biological correlates of suicide have been identified including abnormalities in serotonergic neurotransmission, epigenetic changes and alterations in hypothalamic-pituitary-adrenal axis function. ${ }^{25}$ Only two studies have investigated these phenomena in PD. One was assigned a low-quality rating, and the other a very low quality rating. Neither investigated suicide as a primary outcome and the implications of their findings are unclear. ${ }^{\mathrm{s} 29, \mathrm{~s} 30}$
Table 1 Summary of risk factors for fatal suicide in the general population and their association with fatal suicide in persons with Parkinson's disease ${ }^{13,26,27,54,510,513,531}$

\begin{tabular}{|c|c|c|}
\hline $\begin{array}{l}\text { Risk factors for suicide in the general } \\
\text { population }\end{array}$ & $\begin{array}{l}\text { Studies examining } \\
\text { these risk factors } \\
\text { (quality rating) }\end{array}$ & $\begin{array}{l}\text { Evidence trend } \\
\text { in Parkinson's } \\
\text { disease }\end{array}$ \\
\hline Male gender & $\begin{array}{l}\text { Lee, } 2016 \text { (3); } \\
\text { Myslobodsky, } 2001 \\
\text { (3); Li, } 2018 \text { (1) }\end{array}$ & Increased risk \\
\hline European American ethnicity & $\begin{array}{l}\text { Myslobodsky, } 2001 \\
\text { (3) }\end{array}$ & Increased risk \\
\hline Education level & $\begin{array}{l}\text { Myslobodsky, } 2001 \\
\text { (3) }\end{array}$ & No effect \\
\hline Unemployment & None & Not studied \\
\hline Single/Divorced/Bereaved & $\begin{array}{l}\text { Myslobodsky, } 2001 \\
\text { (3); Li, } 2018 \text { (1) }\end{array}$ & $\begin{array}{l}\text { No effect to } \\
\text { decreased risk }\end{array}$ \\
\hline Comorbid medical illness & Li, 2018 (1) & Decreased risk \\
\hline Depressive disorder & $\begin{array}{l}\text { Lee, } 2016 \text { (3); } \\
\text { Mainio, } 2009 \text { (3); } \\
\text { Myslobodsky, } \\
\text { 2001(3); Li, } 2018 \text { (1) }\end{array}$ & Increased risk \\
\hline Bipolar disorder & $\begin{array}{l}\text { Myslobodsky, } 2001 \\
\text { (3) }\end{array}$ & Increased risk \\
\hline Alcohol/substance use disorder & Mainio, 2009 (3) & Increased risk \\
\hline Psychosis & Lee, 2016 (3) & Increased risk \\
\hline Personality disorder & None & Not studied \\
\hline Anxiety disorder & None & Not studied \\
\hline Multiple psychiatric disorders & None & Not studied \\
\hline Hopelessness & None & Not studied \\
\hline Impulsivity & None & Not studied \\
\hline $\begin{array}{l}\text { History of self-harm or past suicide } \\
\text { attempt }\end{array}$ & Mainio, 2009 (3) & Increased risk \\
\hline Access to lethal means & None & Not studied \\
\hline Stressful life events & None & Not studied \\
\hline Childhood maltreatment/Abuse & None & Not studied \\
\hline Alterations in serotonergic function & None & Not studied \\
\hline $\begin{array}{l}\text { Hypothalamic-pituitary-axis } \\
\text { abnormalities }\end{array}$ & None & Not studied \\
\hline Epigenetic changes & None & Not studied \\
\hline
\end{tabular}

\section{Risk factors for suicidality}

See table 1 for a summary of known suicide risk factors in the general population and the relationship of these risk factors to PwP (as applicable).

\section{Demographics}

Six studies investigated the demographics of suicidality in PwP. One was very low quality, three were low quality and two were moderate quality. As expected based on the demographic profile of PD, suicide victims with PD are older than suicide victims in the general population (average 12.6 years older), but are younger than PwP who die of other causes. ${ }^{\text {s13,s31 }}$ SI was associated with younger age in all but one of the reviewed studies. ${ }^{\mathrm{s}, \mathrm{s} 15, \mathrm{~s} 21, \mathrm{~s} 32}$ PwP who die by suicide have fewer medical comorbidities than those who die of other causes, while the presence of comorbidities increases the risk of SI. ${ }^{s 13, s 31}$ Male gender increases the risk of suicide in PwP, but there are no gender differences in rates of SI. ${ }^{\mathrm{s} 4, \mathrm{~s} 10, \mathrm{~s} 13, \mathrm{~s} 21, \mathrm{~s} 32}$ Caucasian ancestry and being from a rural area are also risk factors for suicide. ${ }^{s 4, s 10, s 13}$ Suicide victims with PD are more likely to be married than suicide victims in the general population, ${ }^{\text {s13 }}$ but marital status appears to have no association with SI. ${ }^{\text {7 }}, \mathrm{s} 21$ The role of level of education in fatal suicide and SI 


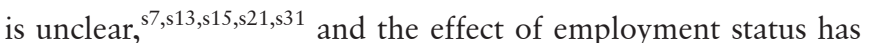
not been studied in this population.

\section{Neuropsychiatric symptoms}

Sixteen studies examined the impact of neuropsychiatric symptoms on suicidality in PwP. Two were assessed to be very low quality, with the rest being low or moderate quality. Two large studies in Northern Finland $(n=555)$ and South Korea $(n=4362)$ found a significant association between suicide in PwP and history of any mental illness. ${ }^{\mathrm{s}, \mathrm{s} 10}$ Depression is nearly invariably associated with SI and SAs in relevant publications, ${ }^{54, \mathrm{~s} 7, \mathrm{~s} 15, \mathrm{~s} 16, \mathrm{~s} 18 \text {-s2 }}$ $1, \mathrm{~s} 32, \mathrm{~s} 33$ with only one study failing to find an association. ${ }^{\mathrm{s} 31}$ SI has been found to be a valid marker of PD depression. ${ }^{\text {s34,s35 }}$

Fewer studies associate psychosis with suicidality in PwP, although one study found the risk of SI may be higher in those with psychosis (OR 19.2; 95\% CI 1.4 to $27.3 ; \mathrm{p}=0.026)$ than with depression (OR 4.6; 95\% CI 2.2 to 9.4 ; $\mathrm{p}<0.001$ ). ${ }^{\mathrm{s}, \mathrm{s} 18, \mathrm{~s} 19}$ Delusions in particular were associated with suicide risk in one large study $(\mathrm{n}=4362) .{ }^{\mathrm{s} 4}$ Anxiety disorders have been correlated with SI in PwP. ${ }^{\mathrm{s} 16, \mathrm{~s} 21}$ Only one study has looked at the role of substance abuse, finding that alcohol use contributed to suicide in PwP at rates similar to the general population. ${ }^{\text {s10 }}$ Importantly, PwP who die by suicide are much more likely to have a past SA compared with suicide victims in the general population $(44 \%$ vs $9.9 \%) .{ }^{s 10}$ Increasing scores on the Beck Hopelessness Scale, Hamilton Depression Rating Scale and Beck Depression Inventory have been associated with SI in PwP. ${ }^{\mathrm{s} 7, \mathrm{~s} 21}$

Suicide attempters with major depression in the general population have been found to have higher levels of impulsivity than those who do not attempt suicide. ${ }^{28} 29$ As such, impulse control disorders (ICDs) are a potentially important contributor to suicide and SI in PwP, especially given that ICDs are correlated with use of dopamine agonists. ${ }^{30}$ Three of the reviewed studies looking at a total of over $300 \mathrm{PwP}$ found a positive correlation between ICDs and SI on univariate but not multivariate analyses. $^{\text {s15,s } 18, \mathrm{~s} 20}$ There were no studies investigating the relationship between ICDs and SAs or deaths by suicide.

\section{Cognition}

Most studies excluded patients with dementia or Mini-Mental State Examination $(\mathrm{MMSE})<24$. Four articles directly investigated cognition. All were low or very low quality and the findings varied. One abstract detailing a cross-sectional study of 1783 PwP found that suicidal PwP were younger (on average 66.6 years vs 69.4 years) but had more cognitive impairment when compared with PwP who were not suicidal. ${ }^{\mathrm{s} 2}$ In contrast, two much smaller studies found an association between suicide or SI and higher MMSE score. ${ }^{\mathrm{s} 7, \mathrm{~s} 31}$ Another found no association. ${ }^{\mathrm{s} 21}$

\section{Motor symptoms and other PD-related variables}

Ten publications examined the effect of motor symptoms and other PD-related variables on suicidality in PwP. All were low or moderate quality, with one study being very low quality. The largest of these studies showed that severity of motor impairment, Hoehn and Yahr (H\&Y) stage and illness duration were not associated with suicide risk, but that suicide was more common in PwP with generalised or upper extremity onset of motor symptoms. ${ }^{44}$ Several smaller studies have found no association between SI and disease severity, stage or duration. ${ }^{\mathrm{s7}, \mathrm{s} 18, \mathrm{~s} 21}$

In contrast, some have reported a correlation between increased motor severity ${ }^{\mathrm{s} 15, \mathrm{~s} 16, \mathrm{~s} 32}$ or longer disease duration ${ }^{\mathrm{s} 11, \mathrm{~s} 15}$ and SI. PwP who die by suicide have also been found to have lower H\&Y stage and Unified Parkinson's Disease Rating Scale
(UPDRS) scores compared with those who die from other causes. ${ }^{\text {s31 }}$ Suicidality has been correlated with bradykinesia, rigidity, axial impairment and posture and gait disturbance items on the UPDRS. ${ }^{\mathrm{s} 3, \mathrm{~s} 37}$ There is limited and conflicting evidence on the impact of earlier disease onset. ${ }^{\mathrm{s} 38, \mathrm{~s} 39}$

Dyskinesias or unpredictable motor OFF periods have not been associated with SI. ${ }^{\text {s7,s18 }}$ Motor fluctuations may be associated with suicidality, especially when patients consider the fluctuations to be inadequately controlled by their current treatment regimen. ${ }^{\mathrm{s} 31, \mathrm{~s} 40}$ It is unclear whether this finding reflects a neurobiological relationship or the need for clinicians to effectively manage expectations as fluctuations inevitably commence. Similarly, there is increasing understanding of the prevalence of non-motor OFF periods or fluctuations in PwP. These are known to have an important impact on quality of life, but have not yet been studied in relation to suicidality. ${ }^{31}$ Tremor and akathisia have not been associated with SI. ${ }^{\mathrm{s} 36, \mathrm{~s} 37, \mathrm{~s} 41}$

\section{Suicide risk compared with other populations}

Eight articles compared the risk of suicide in PwP to individuals with other disorders. Two were moderate quality and the rest were low quality. Three low-quality studies compared the risk of suicide in depressed individuals with and without PD. Merschdorf et $a l^{542}$ looked at depressive symptoms among German inpatients admitted to psychiatric or neurological hospitals. They compared symptoms in 49 depressed PwP with 38 persons with major depression alone. Fewer depressed PwP reported SAs (4\% vs 42\%), despite no difference in the frequency of SI (reported in 76\% of both groups). ${ }^{542}$ Similarly, Zhou et al ${ }^{543}$ reported in an abstract that there were no significant differences in the frequency of SI in depressed individuals with or without PD. Depressed PwP have been found to be at higher risk for suicide than non-depressed PwP or non-depressed individuals without PD after acute myocardial infarction. ${ }^{\text {s3 }}$

There has been limited research comparing suicide risk in PD to other movement disorders. One study found higher rates of suicidality in PwP and multiple system atrophy when compared with individuals with progressive supranuclear palsy, despite similar rates of depression in all three groups. ${ }^{544}$ There appears to be no difference in rates of SI between patients with idiopathic $\mathrm{PD}$, monogenic PD and spinocerebellar ataxia. ${ }^{\mathrm{s} 7, \mathrm{~s} 38}$

In the only study comparing suicidality among PwP to other chronically disabled individuals, $\mathrm{PwP}$ had significantly higher rates of depression and suicidality despite less severe disability. ${ }^{s 45}$

\section{Effects of PD treatments}

Dopamine and dopamine agonists

While clearly invaluable for management of PD overall, the role of dopamine replacement therapy (DRT) in suicide risk is unclear. In the first study to comment on the effect of DRT, Cherington $^{\text {s46 }}$ reported on three PwP who developed suicidality after several months of treatment with levodopa. This led the author to caution that use of levodopa may be limited by severe depressive symptoms including suicidality. ${ }^{546}$ Soon after, two other groups reported cases of suicide in PwP after starting levodopa. ${ }^{\text {s47,s48 }}$

Subsequent studies have been less consistent, possibly due to more sophisticated DRT regimens. Case reports have described suicidality after withdrawal of levodopa or dopamine receptor agonists which resolves when the medications are resumed. ${ }^{\text {s49- }}$ s51 Dopamine agonist withdrawal syndrome (DAWS) is a well described clinical phenomenon associated with dysphoria and other psychiatric symptoms. ${ }^{32}$ Such symptoms could provide a 
mechanism for increased suicide risk. Clinically, the development of suicidality in individuals on 'drug holidays' has been one reason the practice has fallen out of favour, although we did not find specific reference to these 'drug holidays' in our review of the literature.

In seeming contrast to the idea that dopamine withdrawal precipitates suicidality, one group found an association between suicide and higher levodopa dosage which persisted after adjusting for age, sex and other clinical variables. ${ }^{s 4}$ Other reports have found weak or non-statistically significant associations between higher levodopa dose or dopamine agonist use and suicidality. ${ }^{\mathrm{s} 11, \mathrm{~s} 21, \mathrm{~s} 31}$ Only one of these groups looked at rates of ICDs in its participants, but was unable to draw any conclusions about the relationship between ICDs, DRT or dopamine agonist dose, and suicidality as none of the participants had an ICD. ${ }^{\text {221 }}$ Mania in the setting of misuse of levodopa has also led to suicide. ${ }^{552}$

\section{Other medications}

There have been several case reports and one open-label study describing suicide in PwP after starting Duodopa/Levodopa-Carbidopa Intestinal Gel (LCIG). These suicides occurred between 3 months and 5 years after starting LCIG. ${ }^{\text {s26,s53-s57 }}$

Recently, safety and tolerability studies have included the Columbia-Suicide Severity Rating Scale (C-SSRS) in their standard assessment battery. The C-SSRS assesses for SI and suicidal behaviours, including attempts and death by suicide. Tozadenant (a selective adenosine $A_{2 A}$ antagonist effective for motor symptoms in animal models) and the catechol-O-methyl transferase inhibitors opicapone and entacapone have been studied in this manner and have shown no increase in C-SSRS scores. ${ }^{\text {s58-s61 }}$ One study of pardoprunox (a relatively novel dopamine agonist) reported on a suicide in the placebo group, with no suicide-related adverse events in the treatment group. ${ }^{662}$ Two randomised controlled trials (RCTs) and one open-label study have shown no association of transdermal rotigotine with change in C-SSRS score or increased risk of SI. ${ }^{\mathrm{s} 63-\mathrm{s} 65} \mathrm{Li}$ and colleagues ${ }^{\mathrm{s} 31}$ found no association between PD suicide and entacapone, trihexyphenidyl and selegiline. Notably, most of the trials using the C-SSRS to determine suicide risk have been relatively short-term exposures, with the longest trial lasting 1 year.

\section{Deep brain stimulation (DBS) and other procedural interventions}

DBS may be perceived as a risk factor for suicide, and most DBS centres exclude patients with active SI. ${ }^{\mathrm{s} 66-\mathrm{s} 68}$ Despite this, our review showed little agreement in the literature on whether DBS impacts suicidality. Fifty-one studies looked at the effects of DBS and other procedural interventions on the risk of SI or suicide in PwP. Most were low or very low quality. There were 12 moderate quality studies, one high quality and one very high quality study.

In the largest study of the group, Voon and colleagues ${ }^{569}$ conducted a multicentre retrospective investigation into suicide after subthalamic nucleus (STN) targeted DBS. Out of 5311 patients, 24 died by suicide $(0.45 \%)$ and 48 attempted suicide $(0.9 \%)$. Compared with the general population, suicide rates were elevated for the first year postoperatively and remained elevated for 4 years after surgery. Notably, 75\% of attempted and fatal suicides occurred within 17 months postoperatively. A follow-up nested case-control study found that only postoperative depression was significantly associated with suicide or SA after correction for multiple comparisons. In fact, over $40 \%$ of patients had a diagnosis of depression or apathy at the time of SA. Approximately half of those who attempted or died by suicide had expressed SI and a similar number had seen a healthcare provider within a month of death. ${ }^{\text {s69 }}$

In another landmark study, Weintraub et $a l^{570}$ investigated the impact of DBS on SI and suicidal behaviours using retrospectively analysed data from an RCT for efficacy and safety of DBS versus best medical therapy. In the first phase of the trial, they compared rates of SI and suicidal behaviours in 255 PwP undergoing DBS versus best medical therapy. There were no suicidal behaviours in either group. SI was rare, and rates were not significantly different between groups. Several 'proxy symptoms' for SI including anergia, anxiety, depressed mood and hopelessness showed a trend for decreased frequency in the DBS group. In phase two of the trial, the authors randomised 299 patients to receive STN $(n=147)$ or internal globus pallidus (GPi) targeted DBS $(n=152)$. Rates of SI were similar between these two groups, but several 'proxy symptoms' were worse in the STN group. Specifically, patients who underwent STN-DBS reported decreased happiness, increased anger/bitterness and increased fatigue. ${ }^{\text {s70 }}$

There was considerable variability in the findings of the other studies reviewed in this subtopic. Several described new onset SI after STN-DBS..$^{549,571,572}$ Others have found that DBS does not increase suicidality. ${ }^{\mathrm{s} 11, \mathrm{~s} 18, \mathrm{~s} 73-\mathrm{s} 76}$ One retrospective analysis of 113 patients found that most new SI develops within 6 months postoperatively. ${ }^{\text {s7 }}$

Prevalence of suicide in the reviewed literature ranged from $0.5 \%$ to $2 \%$ up to 5 years after STN-DBS. ${ }^{\text {s24,s25,s78-s82 }}$ Only one study $(n=17)$ fell outside this range, reporting a $5 \%$ suicide rate. ${ }^{\mathrm{s} 83}$ In contrast, the reported prevalence of SAs varies widely. An earlier study by Voon and colleagues ${ }^{\mathrm{s} 79}$ found that $1.7 \%$ of 406 PwP undergoing STN-DBS attempted suicide. Two other studies have reported SA rates around or below $1 \% .^{s 84, s 85}$ However, multiple small studies have reported SAs in 3\%-6\% of patients after DBS. ${ }^{\text {s80,s84,s86,s87 }}$ SAs have been reported anywhere from 1 month to 4 years after STN-DBS, with an average of 1 year. ${ }^{\text {s27,s79-s81,s88-s91 }}$ Several studies have reported no SAs or suicides over up to 10 years of follow-up. ${ }^{\text {s92-s95 }}$

Some groups have investigated predictors of suicidality post-DBS. There is some evidence that early-onset PD may be a risk factor, but this is contested. ${ }^{\mathrm{s} 27, \mathrm{~s} 91,596-\mathrm{s} 99}$ History of depression may be important, ${ }^{\mathrm{s} 2}$ and there have been case reports of suicide after DBS in individuals with and without an active depression. ${ }^{\text {s25,449 }}$ Interestingly, two groups have found an increase in SI and SAs after STN-DBS despite improvement or no change in depressive symptoms. ${ }^{\mathrm{s} 88, \mathrm{~s} 89}$ One group created a screening tool to encourage patients to disclose psychiatric symptoms prior to DBS; however, it is unclear whether such an intervention would impact suicide rate post-DBS. ${ }^{\text {s100 }}$ One retrospective review found no predictors of suicide based on pre-operative neuropsychological evaluation. ${ }^{\text {s101 }}$

Changes in DBS settings have also been implicated in suicidality. Some case reports have described SAs after changes in DBS stimulation intensity, ${ }^{s 49,596}$ with inconsistent resolution after the DBS was turned off or reverted to its original settings. ${ }^{.50,5102}$ Case reports have implicated changes in both the left and right STN electrodes. ${ }^{449, s 103}$ Several studies with varying evidence levels have noted that motor outcome after DBS does not appear to impact suicide risk, and in fact, many of the patients who attempt suicide report improvement in motor symptoms after STN-DBS. ${ }^{\text {.27,s49, } 669,884,590, s 104}$ In some cases, the depressive symptoms and suicidality that develops after STN-DBS are described as having an acute onset. ${ }^{\mathrm{s} 25, \mathrm{~s} 49, \mathrm{~s} 103}$ 
Most literature on suicide after DBS is focused on STN-DBS. Few groups have looked at outcomes after GPi-DBS or compared suicidality in patients receiving different types of DBS. One study found no difference in rates of SI in patients undergoing STN versus GPi-DBS. ${ }^{\text {s105,s106 }}$ As noted above, Weintraub and colleagues $^{\text {s70 }}$ also found no statistically significant difference between patients undergoing STN versus GPi-DBS, but did note a worsening of 'proxy symptoms' for suicide in the STN group. There is one case report of a patient developing SI after GPi-DBS; this resolved after stimulation was discontinued. ${ }^{107}$ Another retrospective cohort study described a case of suicide in an individual with early-onset PD after GPi-DBS. ${ }^{\text {s108 }}$

Several authors have suggested that post-DBS suicide risk may be related to postoperative changes in DRT. ${ }^{s 49, s 50, s 104}$ Many of the studies describing increased suicide risk after DBS were conducted at a time when aggressive reduction of DRT, especially dopamine agonists, was advocated after DBS. As mentioned above, DAWS could potentially precipitate suicide in these individuals. In support of this hypothesis, the study by Voon and colleagues $^{\mathrm{s} 69}$ referenced above showed increased suicidality postoperatively in the setting of a 50\%-60\% DRT dose reduction, while the study by Weintraub et $a l^{570}$, published approximately 5 years later did not show an increase in suicidality in the setting of only a $25 \%$ DRT dose reduction after surgery. ${ }^{\text {s69,s70,s87 }}$ This DRT reduction hypothesis would also explain the finding by Weintraub and colleagues ${ }^{\mathrm{s} 70}$ that 'proxy symptoms' for suicide were worse in patients undergoing STN-DBS as compared with those undergoing GPi-DBS, as STN-DBS typically results in a more significant reduction in DRT dose. ${ }^{570}$ Even so, as noted above the role of DRT itself remains unclear ${ }^{\mathrm{s}, \mathrm{s} 49-\mathrm{s} 51}$ and one prospective study reported that reductions in anti-parkinsonian medications were similar between PwP who developed SI after DBS and those who did not. ${ }^{\text {s71 }}$

There is little information on the impact of other procedural treatments. Isolated suicides have been reported after STN gamma knife radiosurgery and pallidotomy. ${ }^{\text {s109-s111 }}$ It is worthwhile to note that individuals undergoing treatment with advanced therapies such as DBS and other interventional procedures may represent a clinically distinct population with a different suicide risk even in absence of these interventions.

\section{Treatment of suicidality}

There have been eight publications on the treatment of suicidal PwP. All were given a very lowquality rating with the exception of one moderate quality study. This abstract by Linse and colleagues $^{\text {s112 }}$ described a randomised, controlled, rater-blinded study looking at group psychoeducation for treatment of PD depression. Primary outcomes included a measure of suicidality. In this study, group psychoeducation provided short term relief of SI without persistent effect 6 months later. ${ }^{\text {s112 }}$ Regarding the lower quality literature, multiple case reports have noted resolution of suicidality with adjustment of DBS settings, although the directionality of this change does not show a consistent pattern and other case reports have conflicted. ${ }^{549,550, s 102}$ Resolution of suicidal depression has been associated with resump-

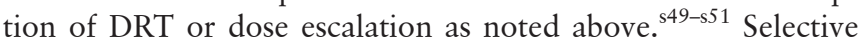
serotonin reuptake inhibitors and serotonin-norepinephrine reuptake inhibitors including fluoxetine, sertraline, escitalopram, mirtazapine and venlafaxine have been used to treat PwP with SI and after SAs. These medications have been used with and without simultaneous changes to DRT. ${ }^{\text {s4-s51,s91,s113 }}$ Quetiapine and clonazepam have also been used in this population. ${ }^{\mathrm{s} 51}$ Case reports have suggested that electroconvulsive therapy may relieve suicidality in depressed PwP, even those with a DBS in place. s51,s102,s114-s116 $^{\text {s. }}$

\section{DISCUSSION}

In this review, we identified 116 papers summarising the prevalence of suicide in PD, demographic profiles of victims, risk factors, the effect of PD treatments including DBS and the treatment of suicidality in PwP. We identified a relative dearth of good quality studies, with the literature primarily limited to case reports, abstracts or small studies in which suicidality was a secondary outcome or incidental finding. There were few large, longitudinal studies and even fewer RCTs.

The current literature is often conflicting. While this is certainly attributable at least in part to the complexity of both PD and suicidality, a number of other factors may also contribute. First, SI or SAs are far more common than suicide and thus easier to study but cannot necessarily be extrapolated to understand fatal suicidal acts. The lack of consistent operationalisation of these terms is also a limitation, along with the lack of validated scales to measure suicidality in PwP.

We found that PwP are much more likely to suffer from SI than the general population. ${ }^{33}$ What remains less clear is whether PwP are more likely to attempt or die by suicide. Prevalence studies vary widely depending on the population. PwP appear to have decreased or unchanged suicide risk in the USA, Denmark, Finland and Canada, while those in South Korea, England, Netherlands and Sweden appear to be at increased risk. Perhaps this is related to general cultural differences or differences in the standard management of PD and PD depression. Future research should also specifically consider the role of gender disparities and ethnic variation in PD suicide.

Like in the general population, depressive symptoms clearly increase the risk of SI and death by suicide in PwP. However, prior SA may be a more significant risk factor for PwP. There have been many studies investigating treatment of SI in the general population and similar studies are greatly needed in the PD population. ${ }^{3435}$ At this time, the literature suggests that aggressive management of depression is the best approach. Importantly, there have been no studies on the impact multidisciplinary care or access to psychiatric treatment may have on suicidality in PwP. Although somewhat controversial, we expected more discussion of assisted-death in the literature reviewed. Assisted-death has been studied in other neurodegenerative disorders and dementia ${ }^{3637}$ and could be an area for future research in PwP.

We were struck by the limited research on access to lethal means and suicide risk in PwP. There has been extensive research on this issue in the general population. ${ }^{15}{ }^{38}$ Elderly men have the highest rate of suicide by firearm, ${ }^{39}$ and the Alzheimer's Association, Veterans Health Administration and others have recommended screening persons with dementia for firearm access. ${ }^{40}$ While the effectiveness of restricting access to lethal means in PwP has not been studied, given shared risk factors it may be prudent to screen PwP similarly and discuss firearm safety early in the disease course. Lethal means counselling may be of particular importance in rural areas, given the outsized role of firearms in rural suicides. ${ }^{41}$

Motor symptoms and other disease-related variables have been studied but evidence remains conflicting. The potential impact of non-motor fluctuations on suicide risk is an important omission from the current body of literature. The impact of DRT on suicidality is also unclear. Some studies suggest dopamine and dopamine agonist withdrawal may precipitate suicide, while 
others show an increased risk of suicidality with higher doses of DRT. The rate of change and the role of 'adequate' versus 'inadequate' DRT have not been studied and could account for some of these discrepancies. Alternatively, DRT may precipitate suicide by increasing mobility and activation in PwP whose depressive symptoms are still inadequately controlled, similar to what has been described with antidepressant initiation in the general population. Likewise, the role of DBS is also poorly understood. The highest quality study to date found no suicidal behaviours and no difference in SI between patients assigned to DBS versus best medical therapy. However, multiple lower quality studies continue to suggest an association.

Perhaps this variability provides evidence for depressive subtypes in PD or a more significant role for apathy, delirium or other neuropsychological changes. Impulsivity is especially important and understudied in PwP given that ICDs may be iatrogenically induced and thus a modifiable risk factor. In the general population with major depressive disorder, specific depressive symptoms have been correlated with a higher risk of suicide including anxiety, ${ }^{42}$ insomnia ${ }^{43}$ and feelings of worthlessness. ${ }^{44}$ Severity of depression has also been associated with increased risk for suicide in the depressed general population. ${ }^{45}$ Association of severity of depression or specific depressive symptoms with suicide, SAs or SI has not yet been studied in PwP, even though, as noted above, PwP may have different depressive profiles than the general population. In the general population, much is known about the specific neuropsychological profile seen in suicidal individuals, such as attentional biases and executive dysfunction. ${ }^{46} 47$ This remains an area in need of study in PwP. Finally, alterations in immune function and serotonin neurotransmission have been implicated in suicide in the general population. ${ }^{25} 48 \mathrm{PD}$ is known to cause aberrations in these systems; however, the impact of such changes on suicide risk has not yet been investigated. ${ }^{49} 50$

Moving forward, it may be important for PD researchers to consider the stress-diathesis model used to explain suicide in the general population. This model proposes that suicidality develops due to an interaction between stressors and the traits which modulate an individual's reaction to those stressors. ${ }^{51}$ While this review highlights the role of stressors in PD suicide, the diathesis component of this model has been largely neglected. This is important, as there is evidence that an individual's perception of a stressor matters more than the stressor itself. ${ }^{52}$ Personality traits are an important contributor to the diathesis component and may be especially important in PwP. For instance, introversion and neuroticism are associated with PD and with suicidal behaviours in the general population. ${ }^{53} 54$ Similarly, there is some evidence that certain factors are protective against suicide in the general population, such as family and moral obligations. ${ }^{52}$ Protective factors were not directly studied in the literature we reviewed, but a better understanding of such factors in PwP could inform suicide prevention efforts. Additional references can be found in the online supplementary file.

Acknowledgements The authors would like to acknowledge Brian Monaghan for his review of the text.

Contributors MDS: conception, organisation, execution, analysis of reviewed studies, drafting the manuscript. Responsible for overall content as the guarantor. KP: conception, analysis of reviewed studies, manuscript review and critique. MPGB, PN: analysis of reviewed studies, manuscript review and critique. JTH, AB, KAM and NMF: conception, manuscript review and critique. JN: conception, organisation, execution, manuscript review and critique. GP: conception, organisation, manuscript review and critique.
Funding The authors have not declared a specific grant for this research from any funding agency in the public, commercial or not-for-profit sectors.

Competing interests KAM receives support from Abbott for research support for his role as site PI for DBS trial, outside the submitted work. There are no other competing interests to report.

Patient consent Not required.

Provenance and peer review Not commissioned; externally peer reviewed.

\section{REFERENCES}

1 Pringsheim T, Jette N, Frolkis A, et al. The prevalence of Parkinson's disease: a systematic review and meta-analysis. Mov Disord. 2014;29:1583-90.

2 Postuma RB, Berg D, Stern M, et al. MDS clinical diagnostic criteria for Parkinson's disease. Mov Disord. 2015;30:1591-601.

3 Duncan GW, Khoo TK, Yarnall AJ, et al. Health-related quality of life in early Parkinson's disease: the impact of nonmotor symptoms. Mov Disord. 2014;29:195-202.

4 Aarsland D, Larsen JP, Tandberg E, et al. Predictors of nursing home placement in Parkinson's disease: a population-based, prospective study. J Am Geriatr Soc 2000;48:938-42.

5 Hughes TA, Ross HF, Mindham RHS, et al. Mortality in Parkinson's disease and its association with dementia and depression. Acta Neurol Scand 2004;110:118-23.

6 Stone DM, Simon TR, Fowler KA, et al. Vital Signs: Trends in State Suicide Rates United States, 1999-2016 and Circumstances Contributing to Suicide - 27 States, 2015. MMWR Morb Mortal Wkly Rep 2018;67:617-24.

7 Centers for Disease Control and Prevention NC for HS, 2017. Underlying cause of death 1999-2016 on CDC wonder online database. CDC wonder online database. Available: http://wonder.cdc.gov/ucd-icd10.html [Accessed 22 Aug 2018].

8 Ciulla L, Lopes Nogueira E, da Silva Filho IG, et al. Suicide risk in the elderly: data from Brazilian public health care program. J Affect Disord 2014;152-154:513-6.

9 Webb RT, Kontopantelis E, Doran T. Suicide risk in primary care patients with major physical diseases. Arch Gen Psychiatry 2012;69:256-64.

10 Hubers AAM, Reedeker N, Giltay EJ, et al. Suicidality in Huntington's disease. J Affect Disord 2012;136:550-7.

11 Christensen J, Vestergaard M, Mortensen PB, et al. Epilepsy and risk of suicide: a population-based case-control study. Lancet Neurol 2007;6:693-8.

12 Harwood D, Hawton K, Hope T, et al. Psychiatric disorder and personality factors associated with suicide in older people: a descriptive and case-control study. Int J Geriatr Psychiatry 2001;16:155-65.

13 Hawton K, van Heeringen K, Suicide vanHK. Suicide. The Lancet 2009:373:1372-81.

14 Bernert RA, Turvey CL, Conwell Y, et al. Association of poor subjective sleep quality with risk for death by suicide during a 10-year period: a longitudinal, populationbased study of late life. JAMA Psychiatry 2014;71:1129-37.

15 Mann JJ, Michel CA. Prevention of firearm suicide in the United States: what works and what is possible. Am J Psychiatry 2016:173:969-79.

16 O'Connor E, Gaynes BN, Burda BU, et al. Screening for and treatment of suicide risk relevant to primary care: a systematic review for the U.S. Preventive Services Task Force. Ann Intern Med 2013;158:741-54.

17 Reijnders JSAM, Ehrt U, Weber WEJ, et al. A systematic review of prevalence studies of depression in Parkinson's disease. Mov Disord Off J Mov Disord Soc 2008;23:183-9. quiz 313. doi.

18 Ehrt U, Brønnick K, Leentjens AFG, et al. Depressive symptom profile in Parkinson's disease: a comparison with depression in elderly patients without Parkinson's disease. Int J Geriatr Psychiatry 2006;21:252-8.

19 Kritzinger C, Vollstedt E-J, Hückelheim K, et al. Qualitative characteristics of depression in parkinson's patients and controls. Behav Neurol 2015;2015:1-5.

20 Gjerstad MD, Wentzel-Larsen T, Aarsland D, et al. Insomnia in Parkinson's disease: frequency and progression over time. J Neurol Neurosurg Psychiatry 2007;78:476-9.

21 Broen MPG, Narayen NE, Kuijf ML, et al. Prevalence of anxiety in Parkinson's disease: a systematic review and meta-analysis. Mov Disord. 2016:31:1125-33.

22 Levac D, Colquhoun H, O'Brien KK. Scoping studies: advancing the methodology. Implementation Sci 2010;5:1-9.

23 Balshem $\mathrm{H}$, Helfand $\mathrm{M}$, Schünemann $\mathrm{HJ}$, et al. Grade guidelines: 3. Rating the quality of evidence. J Clin Epidemiol 2011:64:401-6.

24 Kessler RC, Borges G, Walters EE. Prevalence of and risk factors for lifetime suicide attempts in the National comorbidity survey. Arch Gen Psychiatry 1999:56:617-26.

25 van Heeringen K, Mann JJ. The neurobiology of suicide. The Lancet Psychiatry 2014;1:63-72

26 Yoshimasu K, Kiyohara C, Miyashita K, et al. Suicidal risk factors and completed suicide: meta-analyses based on psychological autopsy studies. Env Heal Prev Med 2008:13:243-56.

27 Nock MK, Borges G, Bromet EJ, et al. Suicide and suicidal behavior. Epidemiol Rev 2008:30:133-54.

28 Coryell W, Wilcox H, Evans SJ, et al. Aggression, impulsivity and inflammatory markers as risk factors for suicidal behavior. J Psychiatr Res 2018;106:38-42.

29 Perroud N, Baud P, Mouthon D, et al. Impulsivity, aggression and suicidal behavior in unipolar and bipolar disorders. J Affect Disord 2011 
30 Grall-Bronnec M, Victorri-Vigneau C, Donnio Y, et al. Dopamine agonists and impulse control disorders: a complex association. Drug Saf 2018;41:19-75.

31 Storch A, Schneider CB, Wolz M, et al. Nonmotor fluctuations in Parkinson disease: severity and correlation with motor complications. Neurology 2013;80:800-9.

32 Rabinak CA, Nirenberg MJ. Dopamine agonist withdrawal syndrome in Parkinson disease. Arch Neurol 2010;67:58-63.

33 Kessler RC, Borges G, Walters EE. Prevalence of and risk factors for lifetime suicide attempts in the National Comorbidity Survey. Arch Gen Psychiatry 1999;56:617-26

34 Hawton K, Witt KG, Taylor Salisbury TL, et al. Pharmacological interventions for selfharm in adults. Cochrane Database Syst Rev 2015;14.

35 Hawton K, Witt KG, Taylor Salisbury TL, et al. Psychosocial interventions for self-harm in adults. Cochrane Database Syst Rev 2016;22.

36 Emanuel EJ, Onwuteaka-Philipsen BD, Urwin JW, et al. Attitudes and practices of euthanasia and physician-assisted suicide in the United States, Canada, and Europe. JAMA 2016;316:79.

37 Draper B, Peisah C, Snowdon J, et al. Early dementia diagnosis and the risk of suicide and euthanasia. Alzheimer's Dement 2010;6:75-82.

38 Anestis MD, Anestis JC, Butterworth SE. Handgun legislation and changes in statewide overall suicide rates. Am J Public Health 2017;107:579-81.

39 Mertens B, Sorenson SB. Current considerations about the elderly and firearms. Am J Public Health 2012;102:396-400.

40 Betz ME, McCourt AD, Vernick JS, et al. Firearms and dementia: clinical considerations. Ann Intern Med 2018:169:47-9.

41 Nestadt PS, Triplett P, Fowler DR, et al. Urban-Rural differences in suicide in the state of Maryland: the role of firearms. Am J Public Health 2017:107:1548-53.

42 Baek JH, Heo JY, Fava M, et al. Anxiety symptoms are linked to new-onset suicidal ideation after six months of follow-up in outpatients with major depressive disorder. $J$ Affect Disord 2015;187:183-7.
43 Pigeon WR, Pinquart M, Conner K. Meta-analysis of sleep disturbance and suicidal thoughts and behaviors. J. Clin. Psychiatry 2012;73:e1160-7.

44 Bolton JM, Belik SL, Enns MW, et al. Exploring the correlates of suicide attempts among individuals with major depressive disorder: findings from the National epidemiologic survey on alcohol and related conditions. J Clin Psychiatry 2008:69:1139-49.

45 Hoertel N, Blanco C, Olfson M, et al. A comprehensive model of predictors of suicide attempt in depressed individuals and effect of treatment-seeking behavior. J Clin Psychiatry 2018;79.

46 Keilp JG, Gorlyn M, Russell M, et al. Neuropsychological function and suicidal behavior: attention control, memory and executive dysfunction in suicide attempt. Psychol. Med. 2013;43:539-51.

47 Richard-Devantoy S, Ding Y, Turecki G, et al. Attentional bias toward suicide-relevant information in suicide attempters: a cross-sectional study and a meta-analysis. Journal of Affective Disorders 2016;196:101-8.

48 Oquendo MA, Sullivan GM, Sudol K, et al. Toward a Biosignature for suicide. Am J Psychiatry 2014;171:1259-77.

49 Hirsch EC, Hunot S. Neuroinflammation in Parkinson's disease: a target for neuroprotection? Lancet Neurol 2009:8:382-97.

50 Politis M, Niccolini F. Serotonin in Parkinson's disease. Behav Brain Res 2015;277:136-45

51 van Heeringen K. Stress-Diathesis Model of Suicidal Behavior. In: Dwivedi Y, ed. The neurobiological basis of suicide. Boca Raton: CRC Press/Taylor \& Francis, 2012.

52 Malone KM, Oquendo MA, Haas GL, et al. Protective factors against suicidal acts in major depression: reasons for living. Am J Psychiatry 2000;157:1084-8.

53 Ortigo KM, Westen D, Bradley B. Personality subtypes of suicidal adults. J Nerv Ment Dis 2009;197:687-94.

54 Santangelo G, Garramone F, Baiano C, et al. Personality and Parkinson's disease: a meta-analysis. Parkinsonism Relat Disord 2018;49:67-74. 